\title{
miR-486 functions as a tumor suppressor in esophageal cancer by targeting CDK4/BCAS2
}

\author{
BAOPING LANG ${ }^{1,2}$ and SONG ZHAO ${ }^{2}$ \\ ${ }^{1}$ Department of Thoracic Surgery, Luoyang Central Hospital Affiliated to Zhengzhou University, \\ Luoyang, Henan 471000; ${ }^{2}$ Department of Thoracic Surgery, The First Affiliated Hospital \\ of Zhengzhou University, Zhengzhou, Henan 450052, P.R. China
}

Received May 13, 2017; Accepted October 20, 2017

DOI: $10.3892 /$ or.2017.6064

\begin{abstract}
Esophageal cancer is a common tumor for which morbidity and mortality are high worldwide. We aimed to study alterations in miR-486 expression in esophageal cancers, and the effect miR-486 on esophageal cancer cell function and behavior. We collected esophageal cancer tissues/corresponding normal tissues from 20 patients and utilized three esophageal cancer cell lines and normal esophageal epithelial cells, and the expression of miR-486, CDK4 and BCAS2 was detected by qRT-PCR. Western blotting was used to detect the expression of CDK4 and BCAS2 protein. Then, we overexpressed miR-486 in esophageal cancer cell line EC9706. A series of cell functional analyses, including cell growth, cell cycle, apoptosis, migration and invasion were performed in esophageal cancer cells using colony formation assay, flow cytometry, Transwell and scratch assays, respectively. DualLuciferase reporter gene assay was used to detect the target genes of miR-486. We found that the expression of miR-486 in esophageal cancer tissues and cell lines was significantly lower than that in the normal tissues and normal esophageal epithelial cell line. Overexpression of miR-486 significantly inhibited the colony formation ability, induced G0/G1 phase arrest and apoptosis and suppressed cell migration and invasion in the EC9706 cells. Using bioinformatics and luciferase reporter assay, we identified that CDK4 and BCAS2 may be target genes of miR-486 and levels of CDK4 and BCAS2 were both significantly higher in the esophageal cancer tissues and cell lines than levels in the normal tissues and cells. Furthermore, knockdown of CDK4/BCAS2 coincided with the suppressive effects of miR-486 in esophageal cancer cells. Expression of apoptotic signaling molecules $\mathrm{p} 21$ and caspase-3 was upregulated in the CDK4/BCAS2-knockdown groups. These results suggest that miR-486 may suppress tumor cell
\end{abstract}

Correspondence to: Dr Song Zhao, Department of Thoracic Surgery, The First Affiliated Hospital of Zhengzhou University, No. 1 Jianshe East Road, Zhengzhou, Henan 450052, P.R. China E-mail: zhaosong_doc@126.com

Key words: esophageal cancer, miR-486, CDK4, BCAS2, cell cycle, apoptosis growth and metastasis in esophageal cancer by targeting CDK4/BCAS2. The newly identified miR-486/CDK4/BCAS2 pathway provides further insight into the development and progression of esophageal cancer, which is of great significance to the early diagnosis and detection of esophageal cancer.

\section{Introduction}

Esophageal cancer, located in the area from the esophagus between the throat and stomach, has become the eighth most commonly diagnosed cancer in the past decade. The 5-year survival rate of esophageal cancer patients is only approximately $17.5 \%$ as reported by data of SEER 18 2004-2010 (1). Based on the location and ancestral precancerous cells, esophageal cancer is mainly divided into two subtypes: adenocarcinoma and squamous cell carcinoma (2,3). Alcohol consumption and smoking are related to both subtypes. Gastroesophageal reflux disease (GERD) and obesity are associated with adenocarcinoma, while nitrosamines and nutritional deficiencies could lead to squamous cell carcinoma (4). In the clinic, surgery, chemotherapy and radiotherapy are commonly used to treat esophageal cancer (5).

It has been reported that mutations of several genes are associated with esophageal cancer, such as p53, FasL and EGFR (6-9). Recent research has demonstrated that miRNAs play essential roles in esophageal cancer (10). MicroRNAs are a class of non-coding RNAs of 17-24 nucleotides, which regulate gene expression by targeting specific mRNAs (11-13). Primary transcripts are firstly cleaved by Drosha and clipped by Dicer (14). MicroRNAs act as oncogenes or tumor-suppressor genes in cancers to participate in processes of tumorigenesis, differentiation and apoptosis $(15,16)$. There are various reports concerning the aberrant expression of microRNAs such as miR-21, miR-127 and miR-377 in esophageal squamous cell carcinoma (17-20). miR-486, located in the 40th intron of the ankyrin-1 gene, was firstly identified in human fetal liver $(21,22)$. It has been reported that aberrant expression of miR-486 is present in several types of cancer including gastric cancer, cutaneous $\mathrm{T}$ cell lymphomas and kidney cancer $(23,24)$. It has been found that miR-486 functions as a tumor suppressor in several types of cancer (25-27). Yet, reports of miR-486 in esophageal cancer are rare. In esophageal cancer tissues, miR-486-5p was found to be suppressed 
and it affected cell proliferation, migration and apoptosis to suppress cancer (28). In esophageal cancer tissues, we found aberrantly downregulated expression of miR-486.

In the present study, we investigated the miR-486 expression alteration between esophageal squamous carcinoma and normal tissues and assayed the effects of miR-486 overexpression on esophageal cancer cell proliferation, invasion and apoptosis. Based on the findings of the function of miR-486 in the present study, we conclude that miR-486 plays an essential role in the progression of esophageal cancer and may be a potential therapeutic target for esophageal squamous carcinoma.

\section{Materials and methods}

Sample collection. A total of 20 histopathologically confirmed esophageal squamous carcinoma and corresponding normal samples were collected from patients. All the procedures were approved by the institutional review boards of the participating hospitals affiliated with Zhengzhou Univeristy. The fresh specimens were obtained and stored at $-80^{\circ} \mathrm{C}$ immediately. All specimens were obtained by surgical resection, fixed in $10 \%$ neutral formalin and embedded in paraffin. After hematoxylin and eosin (H\&E) staining, the samples were pathologically diagnosed under a microscope according to the criteria for diagnosis and treatment of esophageal cancer (2011) (29). Relevant clinical pathological information was obtained from the hospital.

Cell culture. Three esophageal cell lines (KYSE150, EC9706 and TE-9) and human normal esophageal epithelial cell line Het-1A were purchased from the Cell Bank of Shanghai Institute of Cell Biology (Chinese Academy of Medical Sciences, Shanghai, China). KYSE150, EC9706 and TE-9 cells were cultured in RPMI-1640 medium (Gibco, Carlsbad, CA, USA) supplemented with $10 \%$ fetal bovine serum (FBS; Gibco) and a $1 \%$ penicillin (Invitrogen, Shanghai, China). Het-1A cells were cultured in Dulbecco's modified Eagle's medium (DMEM; Gibco) supplemented with 10\% FBS and $1 \%$ penicillin. All the cell lines were grown at $37^{\circ} \mathrm{C}$ in an incubator.

$H \&$ E staining of tissue sections. Before staining, the tissues were sliced into 5- $\mu \mathrm{m}$ thick sections and dewaxed in xylene, and rehydrated through a serial of decreasing concentrations of ethanol. After washing in phosphate-buffered saline (PBS), the sections were stained with H\&E. Finally, the sections were dehydrated in increasing concentrations of alcohol and xylene.

$R T-P C R$. Total RNA was extracted from the samples and cell lines using TRIzol reagent (Takara Bio, Shiga, Japan). cDNA of miRNA was synthesized by miRNA cDNA Synthesis kit (Abm Canada Inc., Milton, ON, Canada). cDNA from mRNA was synthesized by RT Master Mix (Abm Canada). The RT-PCR was performed using SYBR ${ }^{\circledR}$ Green Master Mixes (SR1110; Thermo Fisher Scientific, Darmstadt, Germany) according to the manufacturer's instructions. The primers were: GAPDH forward, 5'-TGTTCGTCATGGGTGTGA AC-3' and reverse, 5'-ATGGCATGGACTGTGGTCAT-3'; CDK4 forward, 5'-TGACATTCCCCTCCCACCTCTCC-3' and reverse, 5'-ATCCTCCTGCCTCAGTCTCCCAAGTA-3';
BCAS2 forward, 5-AAGGACAACAGCATCTTCCCAAA AC-3' and reverse, 5'-TCACATACATCTAGTTCATCTACC TAAAGTGTTC-3'; miR-486-5p forward, 5'-ACACTCCAG CTGGGTCCTGTACTGAGCTGCCC-3' and reverse, 5'-CT CAACTGGTGTCGTGGAGTCGGCAATTCAGTTGAGC CCCGAG-3'; U6 forward: 5'-CTCGCTTCGGCAGCACA-3' and reverse, 5'-AACGCTTCACGAATTTGCGT-3'. Relative gene expression was calculated by the $2^{-\Delta \Delta \mathrm{Ct}}$ method.

Western blotting. The tissues and cells were lysed with RIPA buffer supplemented with phenlymethanesulfonyl fluoride (PMSF). After the concentration was quantified, the proteins were subjected to SDS-PAGE and transferred to polyvinylidene difluoride membranes. The membranes were immunoblotted with the primary antibodies: CDK4 (1:500, ab108357; Abcam, Cambridge, UK), BCAS2 (1:500, ab151293; Abcam), GAPDH (1:500, ab8245; Abcam), p21 (1:500, ab109520; Abcam) and caspase-3 (1:300, ab2171; Abcam) overnight at $4^{\circ} \mathrm{C}$. After being rinsed with TBST, the membranes were incubated with secondary antibodies (at a dilution of 1:5,000) conjugated to horseradish peroxidase. The protein bands were visualized and exposed to X-ray film. GAPDH was used as the internal control.

Immunohistochemistry. The sections were rehydrated in xylene and decreasing concentrations of alcohol. Then, the slices were incubated with $3 \% \mathrm{H}_{2} \mathrm{O}_{2}$ for blocking and incubated with $10 \mathrm{mM}$ citric acid for antigen retrieval. After a second blocking in TBS with 5\% BSA, the sections were incubated with the primary antibody CDK4 (1:500) or BCAS2 (1:500), at $4^{\circ} \mathrm{C}$ overnight. Subsequently, the sections were treated with the secondary antibody for $60 \mathrm{~min}$ at room temperature (RT). Finally, a colorizing reagent was used to stain and hematoxylin was used to counterstain cell nuclei.

miRNA/siRNA synthesis and transfection. miR-486 mimics/ NC, target siRNA and control siRNA were synthesized in Shanghai GenePharma Co., Ltd. (Shanghai, China). Cells were transfected with siRNA diluted by Opti-MEM I Reduced Serum Medium using Lipofectamine 2000 (Abm Canada) according to the manufacturer's protocol.

Colony formation assay. After transfection with the miR-486 mimics/NC, the cells were cultured for $48 \mathrm{~h}$ and collected. The cell density was adjusted to 2000 cells/ml and then the cells were seeded in 6-well plates. The cells were observed under a microscope each day and medium was changed every two days. After fixation with $4 \%$ paraformaldehyde, the cells were strained using crystal violet. After washing with PBS, the number of cell colonies was counted for data analysis.

Scratch wound healing assay. After transfection with the miR-486 mimics/NC, the cells were cultured overnight. Cell scratches were made with 1-ml tips and the width of the scratch wounds were kept the same. After washing with PBS, the cells were cultured in an incubator and images of the scratch wounds were captured after $48 \mathrm{~h}$.

Flow cytometry. The cells were cultured for $48 \mathrm{~h}$ and fixed in ice-cold $70 \%$ ethanol for $12 \mathrm{~h}$. After washing, the cells were 


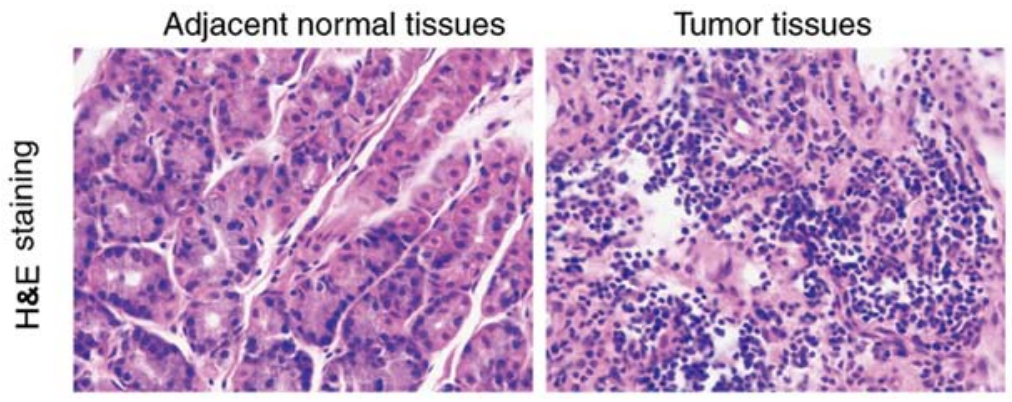

Figure 1. H\&E staining of esophageal squamous cell carcinoma tissue and the adjacent tissue.

incubated with $0.25 \mathrm{mg} / \mathrm{ml} \mathrm{RNase}$ at $37^{\circ} \mathrm{C}$ for $30 \mathrm{~min}$. The cells were resuspended in PI solution $(50 \mu \mathrm{g} / \mathrm{ml})$ and subjected to cell cycle analysis by flow cytometric analysis.

The cells were incubated for $48 \mathrm{~h}$, cells were washed and resuspended in binding buffer and incubated with FITCAnnexin V (Nanjing KeyGen Biotech Co., Ltd., Nanjing, China). After $15 \mathrm{~min}, 5 \mu \mathrm{l}$ PI and $300 \mu \mathrm{l}$ were added to each sample. The cells were incubated for $30 \mathrm{~min}$ at RT in the dark and subjected to flow cytometric analysis within $1 \mathrm{~h}$.

Transwell invasion assay. Transwell assay was performed to detect the invasive ability of the cells. The upper chambers of Transwell plates (BD Biosciences, San Jose, CA, USA) were coated with Matrigel and $\sim 5 \times 10^{4}$ transfected cells were seeded into the chambers. After a 48-h incubation, the cells on the upper chambers were wiped off and the cells on the lower surface were washed. After being fixed, the cells on the lower surface of the membrane were stained with crystal violet to test the extent of invasion.

Dual-Luciferase experiment. The cells were cultured at $60-80 \%$ confluence in $24-w e l l$ plates for $12 \mathrm{~h}$. The reporter plasmids and miR-486-5p were transiently transfected into the cells. After $48 \mathrm{~h}$, the Dual-Luciferase reporter assay (Promega, Madison, WI, USA) was used to measure the Renilla luciferase activity and the data were normalized with firefly luciferase.

Statistical analysis. The Spearman's rank correlation coefficient was used to analyze the ranked data and the Student's t-test was performed to analyze the RT-PCR data. One-way ANOVA was used to analyze the multiple group comparisons. $\mathrm{p}<0.05$ was considered indicative of statistical significance. Each sample was assayed at least three times.

\section{Results}

H\&E staining of esophageal squamous carcinoma tissues and adjacent tissues. According to histological type, esophageal cancer can be divided into esophageal squamous cell carcinoma and adenocarcinoma. Compared to the normal tissues, cancer cells are larger in size than normal cells and the morphology is not consistent. It was observed that giant nuclei, dual nuclei, multi nuclei or heteromorphic nuclei were present in the cancer cells. The ratio of the nucleus and cytoplasm of the cancer cells was different from that of the normal cells (Fig. 1).
Expression of miR-486, CDK4 and BCAS2 in esophageal squamous cell carcinoma tissues and cells. miR-486 expression was significantly downregulated in esophageal squamous carcinoma tissues compared to the corresponding esophageal normal tissues of 20 esophageal cancer patients (Fig. 2A, $\mathrm{p}<0.0001)$. The mRNA and protein levels of CDK4 and BCAS2 were upregulated in the esophageal cancer tissues (Fig. 2A and B, p<0.001). We selected three esophageal cancer cell lines, KYSE150, EC9706 and TE-9, and a normal esophageal epithelial cell line Het-1A. miR-486 exhibited decreased expression while CDK4 and BCAS2 had increased mRNA and protein levels in the three esophageal cancer cell lines than that in the normal cells (Fig. 3 and B).

Through immunohistochemical staining, it was observed that CDK4 and BCAS2 exhibited stronger staining in esophageal squamous carcinoma tissues compared to that noted in the normal tissues (Fig. 2C). The expression of CDK4/BCAS2 was increased in the cell nucleus and cytoplasm.

Overexpression of $\mathrm{miR}-486$ inhibits the colony formation of EC9706 cells. According to the above-mentioned results, the expression of miR-486, CDK4 and BCAS2 in the three esophageal cancer cell lines was the same, thus EC9706 cells were selected for the following experiments. The cells were divided into two groups: NC- and miR-486 mimictransfected EC9706 cells. RT-PCR was performed to detect the level of miR-486 (Fig. 4A). In order to observe whether miR-486 affects the function of EC9706 cells, we detected the cell viability of the two cell groups. The results showed that the colony formation ability was significantly inhibited in the miR-486 mimic-transfected EC9706 cells; the number of colonies in the experimental group was significantly lower than that in the negative control (NC) group (Fig. 4B, p<0.001). These results suggest that $\mathrm{miR}-486$ has the ability to inhibit the colony formation of esophageal cancer cells.

Overexpression of miR-486 induces cell cycle arrest and apoptosis of EC9706 cells. Flow cytometry is a method by which to measure the relative content of DNA, which confirms the cell proportion in each stage of the cell cycle and the apoptotic rate. In order to observe the effects of miR-486 on cell proliferation and apoptosis of the EC9706 cells, we analyzed the percentage of cells in the different stages of the cell cycle and apoptosis using flow cytometry. The results showed that compared with the NC group, overexpression of miR-486 significantly reduced EC9706 cell proliferation, which was 


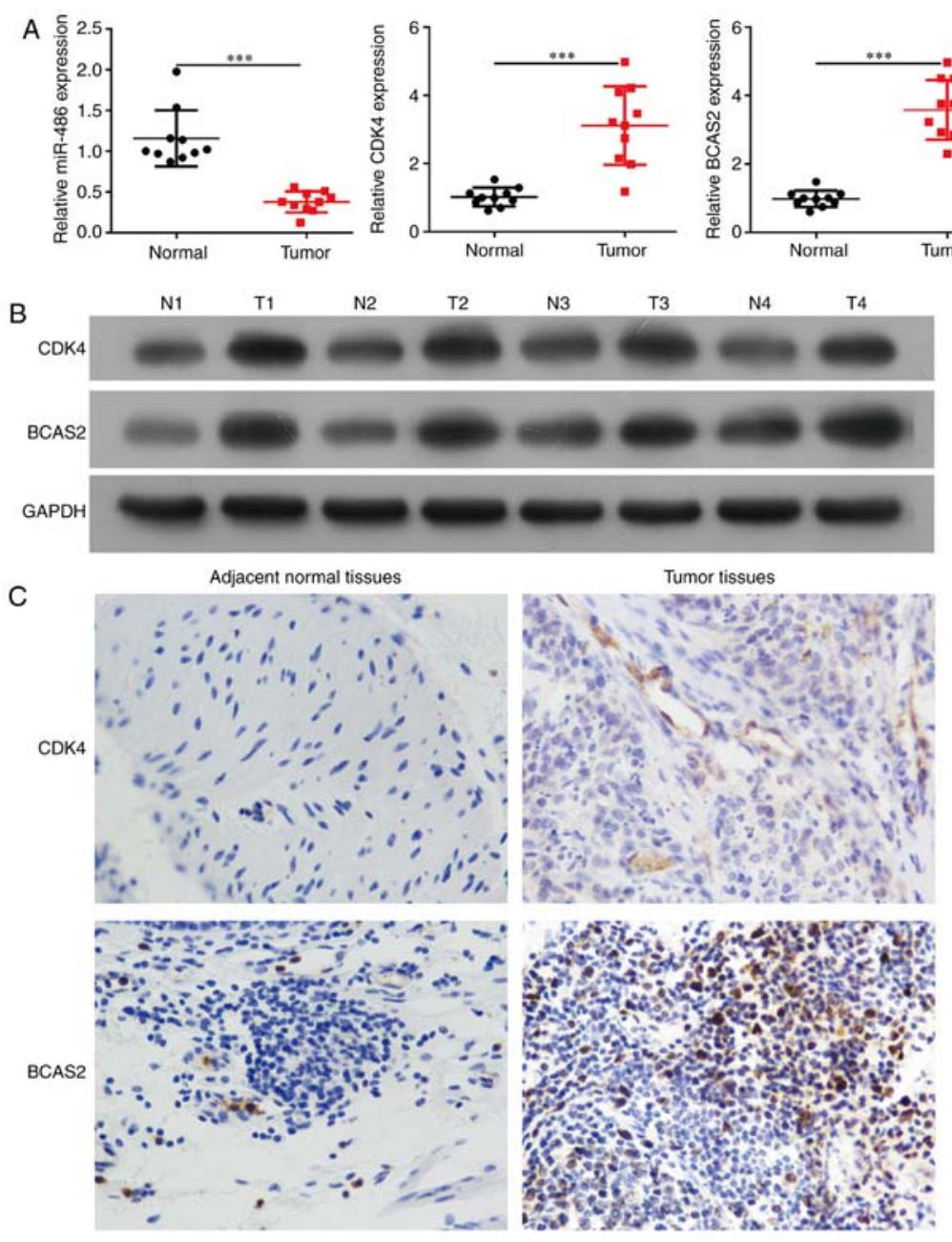

Figure 2. miR-486 expression was significantly downregulated in esophageal squamous carcinoma tissues compared to that noted in the corresponding esophageal normal tissues from 20 esophageal squamous cell carcinoma patients while the expression of CDK4 and BCAS2 was upregulated. (A) The mRNA expression of miR-486, CDK4 and BCAS2 ( $\left.{ }^{* * *} \mathrm{p}<0.001\right)$. (B) Protein levels of CDK4 and BCAS2. (C) Immunohistochemical staining of CDK4 and BCAS2 in esophageal squamous carcinoma tissues and controls.

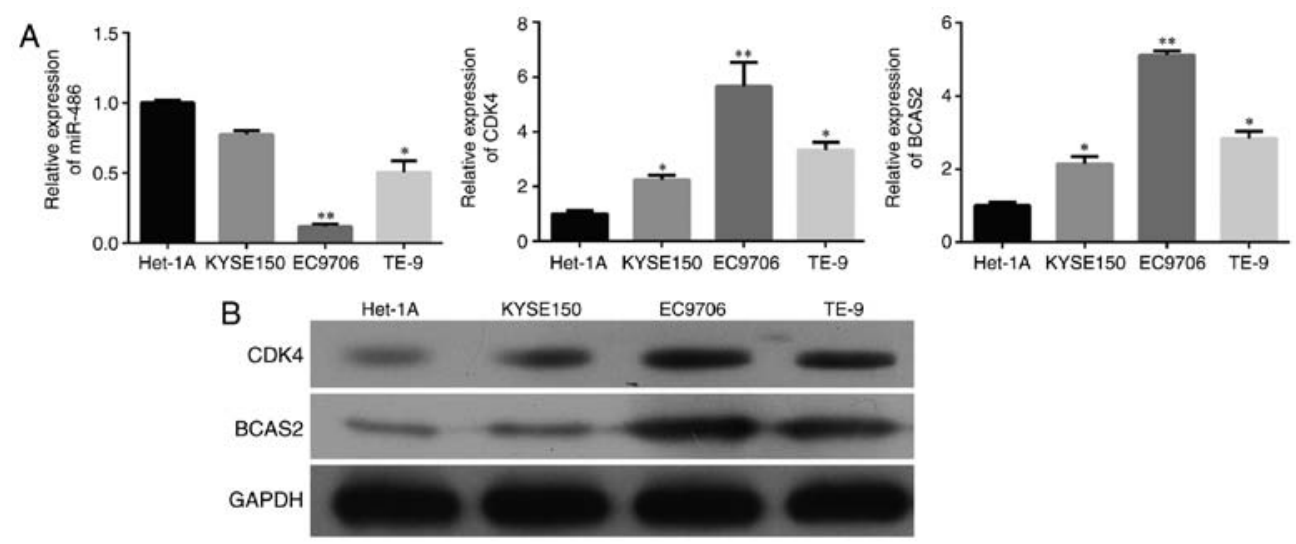

Figure 3. miR-486 expression was significantly downregulated while expression of CDK4 and BCAS2 was upregulated in esophageal cancer cell lines. (A) The mRNA expression of miR-486, CDK4 and BCAS2 ( $\left.\mathrm{p}<0.05 ;{ }^{* *} \mathrm{p}<0.0\right)$. (B) The protein level of CDK4 and BCAS2. P $<0.001$ vs. controls.

mainly reflected by the fact that a higher percentage of cells was arrested in the $\mathrm{G} 0 / \mathrm{G} 1$ phase $(\mathrm{p}<0.05)$. The corresponding proportion of $\mathrm{S}$ phase cells was significantly decreased $(\mathrm{p}<0.01)$, and cells in the $\mathrm{G} 2 / \mathrm{M}$ phase were slightly changed (Fig. 4C).
The change in anti-apoptosis ability is an important characteristic of tumor cells. After overexpression of miR-486 in EC9706 cells, flow cytometry was used to observe the proportion of apoptosis of the two groups of cells. The results showed that the miR-486 mimic-transfected EC9706 cells 

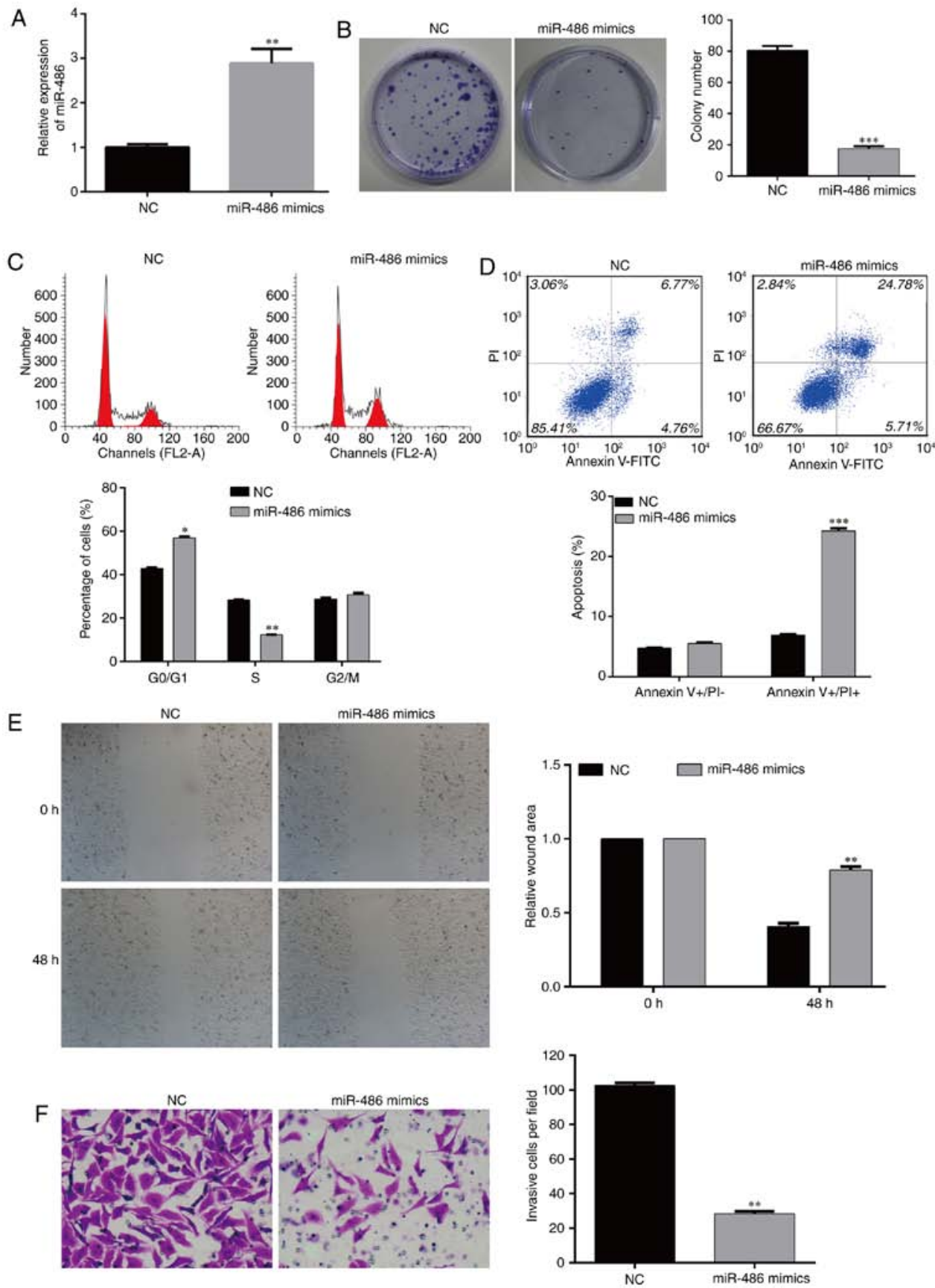

Figure 4. Effects of miR-486 overexpression on the behavior of esophageal cancer EC9706 cells. (A) mRNA level of miR-486 was significantly decreased in the EC9706 cells transfected with the miR-486 mimic compared to the negative control (NC) $\left({ }^{* *} \mathrm{p}<0.01\right)$. (B) Cell colony formation $\left({ }^{* * * *} \mathrm{p}<0.001\right)$. (C) Cell cycle analysis $\left({ }^{*} \mathrm{p}<0.05,{ }^{* *} \mathrm{p}<0.01\right)$. (D) Apoptosis $\left({ }^{* * * *} \mathrm{p}<0.001\right)$. (E) Cell migration $\left({ }^{* *} \mathrm{p}<0.01\right)$. (F) Cell invasion $\left({ }^{* *} \mathrm{p}<0.01\right)$.

had a significantly higher percentage of apoptosis compared with the NC group (Fig. 4D, p<0.001). These results suggest that miR-486 inhibits the cell cycle progression and induces apoptosis in esophageal cancer cells.

Overexpression of miR-486 suppresses the migration and invasion of EC9706 cells. The migration and invasion ability of cancer cells is an important factor related to tumor metastasis. Cell scratch assay was used to observe the motile ability of the cells in the miR-486 mimic-transfected and NC-transfected EC9706 cells. After $48 \mathrm{~h}$, the migration ability of the EC9706 cells with overexpression of miR-486 was significantly lower than that of the NC group, which indicated that miR-486 could inhibit the motility of esophageal cancer cells (Fig. 4E, $\mathrm{p}<0.01)$.

Transwell assay was performed to test the effects of miR-486 on the invasion ability of cancer cells. After overexpression of miR-486 in EC9706 cells, the number of cells invading the basement membrane was significantly lower than that in the NC group (Fig. 4F, p<0.01). These results indicate that miR-486 has the function of reducing the motility and invasion of esophageal cancer cells.

miR-486 regulates the expression of CDK4 and BCAS2. Bioinformatic method was used to predict the binding sites of miR-486 and CDK4/BCAS2 (Fig. 5A). Dual-Luciferase 
A

\begin{tabular}{lll}
\hline & $\begin{array}{c}\text { Predicated consequential pairing of target region (top) } \\
\text { and miRNA (bottom) }\end{array}$ \\
\hline Position 258-264 of CDK4 3'UTR & $5^{\prime}$ & ...UGGGGUCCUUUUUUAUACAGGAA... \\
hsa-miR-486-5p & $3^{\prime}$ & ...GAGCCCCGUCGAGUCAUGUCCUA \\
Position 332-339 of BCAS2 3'UTR & $5^{\prime}$ & ...UAAAGCUUUCAACAUGUACAGGA... \\
hsa-miR-486-5p & $3^{\prime}$ & GAGCCCCGUCGAGUCAUGUCCU \\
\hline
\end{tabular}

B
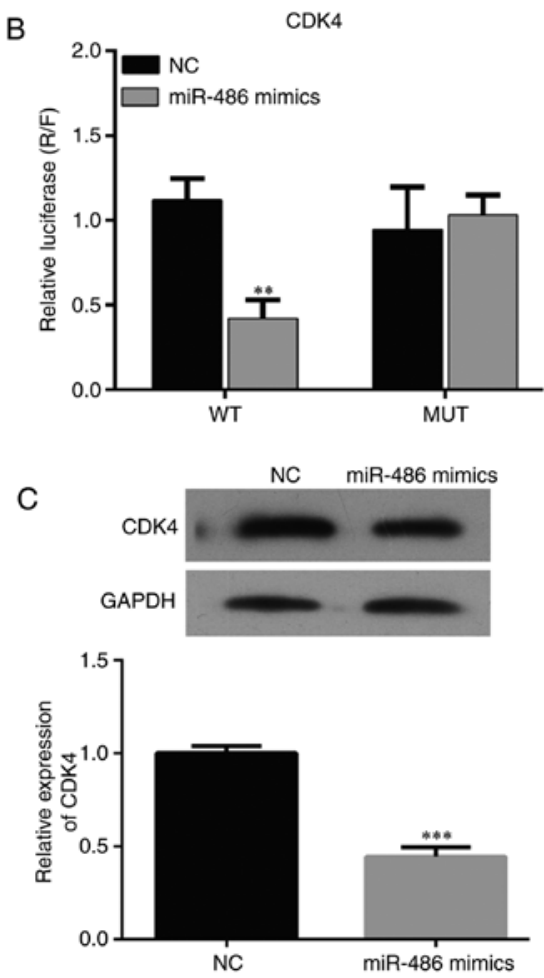

BCAS2
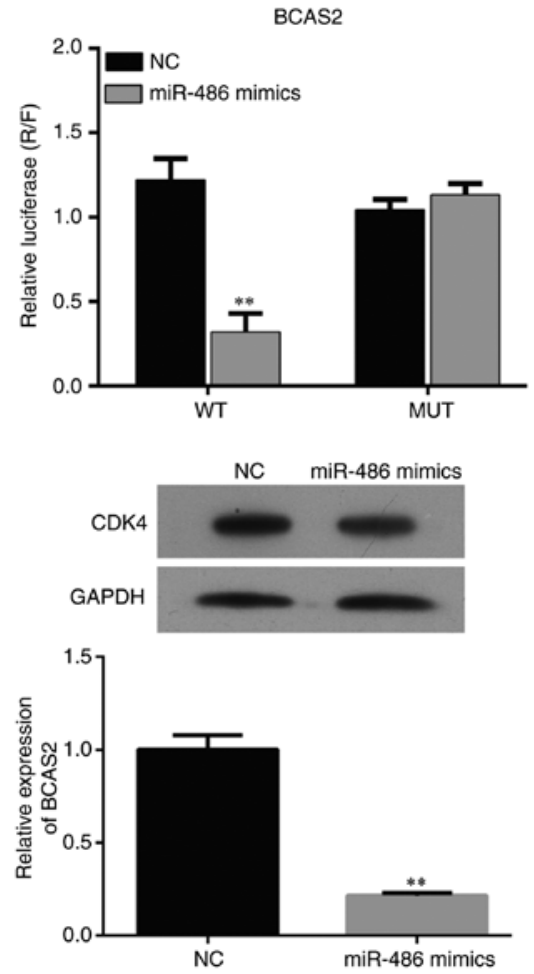

Figure 5. Overexpression of miR-486 suppresses the expression of CDK4 and BCAS2. (A) Predicted consequential pairing of miR-486 and CDK4/BCAS2. (B) Dual-Luciferase assay ( $\left.{ }^{* *} \mathrm{p}<0.01\right)$. (C) Expression of CDK4/BCAS2 was suppressed in the miR-486 mimic-transfected EC 9706 cells $\left({ }^{* * *} \mathrm{p}<0.001,{ }^{* *} \mathrm{p}<0.01\right)$.

assay was used to detect the interaction between miR-486 and CDK4/BCAS2. The results showed that expression of CDK4/ BCAS2 was significantly suppressed in the miR-486 mimictransfected EC9706 cells. When the binding site was mutated, the interaction relationship disappeared and the expression of $\mathrm{CDK} 4 / \mathrm{BCAS} 2$ returned to normal, which indicated that miR-486 may be a regulatory factor of the CDK4/BCAS2 sequence (Fig. 5B and C).

Knockdown of CDK4/BCAS2 downregulates the colony formation of EC9706 cells. In order to study the effects of target genes CDK4/BCAS2 on the behavior of esophageal cancer EC9706 cells, we designed and synthesized siRNAs, siCDK4 and siBCAS2. The effects of these two target genes on cell proliferation and colony formation were detected following transfection. The cells were divided into three groups: NC, siCDK4 and siBCAS2 EC9706 cells. SiCDK4 and siBCAS2 effectively inhibited the expression of the target genes, and the mRNA levels were significantly lower than levels noted in the NC group (Fig. 6A, p<0.01). After the three cell groups were cultured for $48 \mathrm{~h}$, the number of colonies was counted (Fig. 6B). Compared with the NC group, the cell colony formation ability of the siCDK4 and siBCAS2 EC9706 cells was significantly inhibited $(\mathrm{p}<0.05 ; \mathrm{p}<0.01)$.

Knockdown of CDK4/BCAS2 inhibits EC9706 cell cycle progression and induces cell apoptosis. Compared with the NC group, the cell cycle progression of the siCDK4 and siBCAS2 EC9706 groups was significantly blocked. The percentages of cells in the G0/G1 phase of the two target gene-knockout groups were increased significantly, while the proportions of cells in the $S$ phase and G2/M phase were decreased significantly. The cell cycle was arrested in the G0/ G1 phase following the silencing of CDK4 or BCAS2, and could not enter the cell cycle normally (Fig. 6C).

Compared with the NC group, the percentages of apoptotic cells in the target gene knockout groups were increased significantly (Fig. 6D, p<0.01). These data suggest that knockdown of target genes CDK4/BCAS2 can inhibit the cell cycle progression and induce the apoptosis of esophageal cancer cells. 

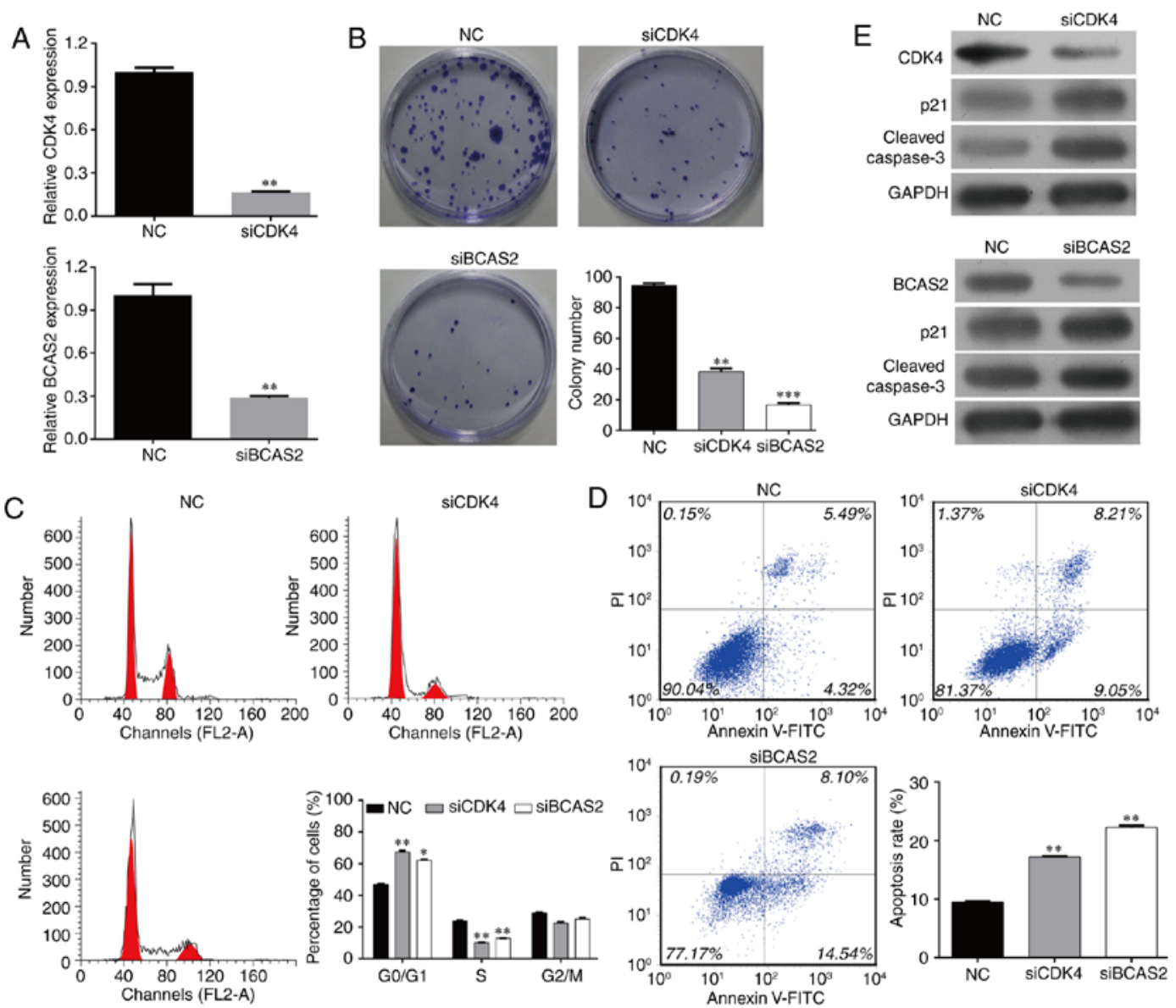

Figure 6. Effects of the knockdown of CDK4/BCAS2 on cell behaviors of esophageal cancer cells. (A) The mRNA levels of CDK4/BCAS2 in the siCDK4 and siBCAS2 EC9706 cell lines ( $\left.{ }^{* *} \mathrm{p}<0.01\right)$. (B) Cell colony formation $\left({ }^{* *} \mathrm{p}<0.05 ;{ }^{* * *} \mathrm{p}<0.01\right.$. (C) Cell cycle ( $\left.\mathrm{p}<0.05,{ }^{* * *} \mathrm{p}<0.01\right)$. (D) Apoptosis $\left({ }^{* *} \mathrm{p}<0.01\right)$. (E) The expression of apoptosis related proteins.
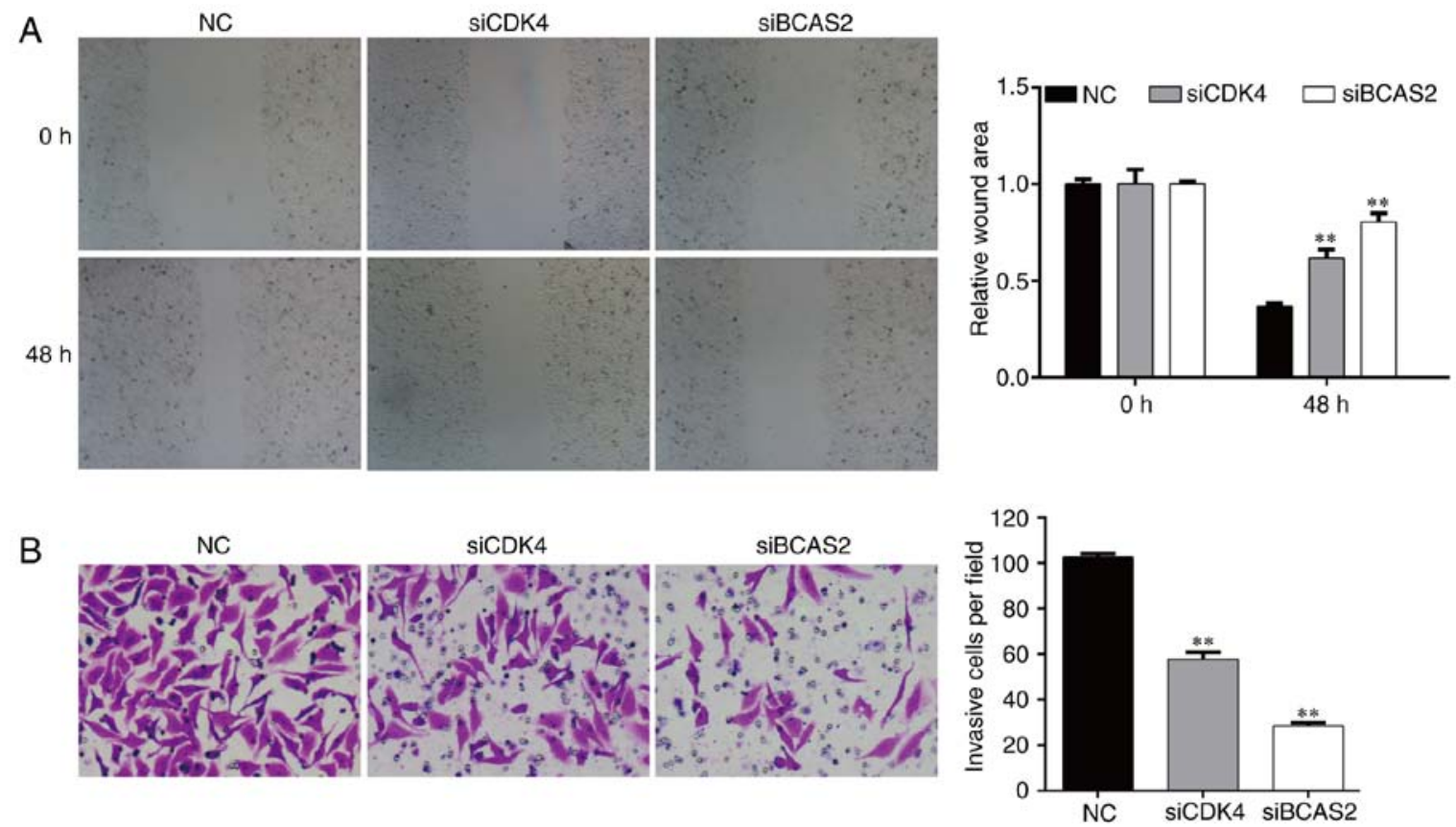

Figure 7. Effects of the knockout of CDK4/BCAS2 on migration and invasion of esophageal cancer cells. (A) Migration ( $\left.{ }^{* *} \mathrm{p}<0.01\right)$. (B) Invasion $(* * \mathrm{p}<0.01)$.

Knockout of CDK4/BCAS2 affects expression of apoptosisrelated proteins. The above results showed that the knockdown of target genes CDK4/BCAS2 can induce the apoptosis of esophageal cancer cells, but the apoptosis-related downstream 
proteins were not clear. In order to study the downstream molecular changes in the apoptotic signaling pathway, the protein levels of p21 and caspase-3 were detected by western blotting. The results showed that compared with the NC group, CDK4 and BCAS2 protein expression was decreased, and expression levels of apoptotic signaling molecules p21 and caspase-3 were also downregulated in the siCDK4 and siBCAS2 EC9706 cell groups (Fig. 6E).

Knockdown of CDK4/BCAS2 inhibits the invasion and migration abilities of EC9706 cells. After transfected for $48 \mathrm{~h}$, the migration ability of the EC9706 cells following silencing of CDK4/BCAS2 was significantly lower than that noted in the NC group (both $\mathrm{p}<0.01$ ), which indicated that knockdown of target genes CDK4/BCAS2 could inhibit the motility of esophageal cancer cells (Fig. 7A).

After knockdown of target genes CDK4/BCAS2 in the EC9706 cells, the number of cells invading the basement membrane was significantly lower compared with that of the $\mathrm{NC}$ group (Fig. 7B, p<0.01). These results suggest that knockdown of target genes CDK4/BCAS2 can reduce the migration and invasion abilities of the esophageal cancer cells.

\section{Discussion}

Esophageal cancer is a common tumor, for which the incidence differs according to race and geographical region. It has been reported that the mortality and morbidity of esophageal cancer is increasing yearly (30-32). Squamous cell carcinoma is one of the main pathological types of advanced esophageal carcinoma. Populations in some regions of northern China have a higher risk of esophageal cancer and esophageal squamous cell carcinoma is the main pathological type, accounting for more than $90 \%$ of all cases (33). miRNAs are a type of non-coding single-stranded RNAs, ranged from 20 to $25 \mathrm{nt}$, and regulate the expression of target genes through interaction with the mRNA 3'-UTR of genes (34). It has been reported that miRNAs are critical diagnostic and prognostic biomarkers in cancer $(35,36)$. miRNAs can function as oncogenes or as tumor-suppressor genes, which play an important role in angiogenesis and epithelial-mesenchymal transition and drug resistance in cancer (37). In the present study, we found that miR-486 exhibited decreased expression in esophageal squamous carcinoma tissues and cell lines compared to that noted in normal tissues and cells. In recent studies, it was found that miR-486 functions as a tumor suppressor in breast, lung and hepatocellular cancer (25-27). In esophageal cancer tissues, miR-486-5p was found to be suppressed and exhibited an anti-oncogene function by affecting proliferation, migration and apoptosis (28). In non-small cell lung cancer, miR-486-5p significantly inhibited cell growth and cell cycle progression by targeting CDK4. The 3'-UTR of CDK4 is the direct interaction region between miR-486-5p and CDK4 (38). In non-small cell lung cancer, miR-486-5p inhibited the progression and metastasis by targeting ARHGAP5 (26). In breast cancer cells, miR-486-5p exerted an anti-proliferative function by targeting PIM-1, in addition to the fact that miR486-5p inhibited proliferation by interacting with PIK3R1 in hepatocellular carcinoma $(25,27)$. However, the function of miR-486 has yet to be elucidated (27). The expression of miR-486 was determined in three esophageal squamous carcinoma cell lines and that of EC9706 had a significantly lower level compared to Het-1A (human esophageal epithelial cell line). Thus, we performed subsequent experiments using EC9706. The differential levels of miR-486 may be due to the difference in cell lines. In our results, overexpression of miR-486 inhibited the ability of colony formation, arrested cell cycle progression and induced apoptosis and its targets were CDK4 and BCAS2. CDK4 (cyclin-dependent kinase 4), a member of the CDK family, is a type of serine/threonine kinase and is an essential signaling transduction molecule which participates in the cell cycle and apoptosis by binding with cyclin proteins. In the progression of the cell cycle, the cyclin proteins are periodically expressed and degraded, and the transient activation of CDK is used to catalyze the phosphorylation of different substrates (39). The CDK family includes CDK1-13 and the cyclin proteins are divided into cyclin A-L, in which cyclin D includes cyclin D1, D2 and D3 $(40,41)$. Cyclin D is expressed in the G1 phase of the cell cycle, binds and activates CDK4 and CDK6, which form the binding complex leading to a series of substrate phosphorylation $(42,43)$. Downstream activated E2F induces a series of protein to transcript and promotes cells enter into the $\mathrm{S}$ phase (44). In the meanwhile, the complex of CDK-cyclin is negatively regulated by CKIs (cyclin kinase inhibitors) (45).

BCAS2 (breast cancer amplified sequence 2), located on chr1 p13.3-21, is a subunit of the prp19 complex, which is an essential factor for the splicing of the cell nuclear precursor mRNA $(46,47)$. BCAS2 is mainly located in the nucleus and has key roles in mitotic initiation (48). Knockdown of BCAS2 using RNAi was found to lead to inhibition of the expression of the other three subunits of PRP19, and the structure of the whole PRP19 complex is shaken, which leads to abnormal mitosis (46-48). In the present study, we found that the proliferation and clone formation ability of esophageal cancer cells were inhibited after knockdown of CDK4 and BCAS2 genes, and the ability of migration and invasion was also reduced. This indicates that a low level of miR-486 leads to the imbalance of CDK4 and BCAS2 in the process of esophageal carcinogenesis, and abnormal increased CDK4 or BCAS2 can promote the esophageal cancer cells to proliferate aberrantly. In addition, p53 is also a target protein of BCAS2, while knockdown of BCAS2 can enhance p53-induced apoptosis (49). These data are consistent with our research. Knockdown of CDK4 and BCAS2 increased levels of the apoptosis-related protein p21 and caspase-3, suggesting that the apoptosis signaling pathway is activated, and the cells are induced to enter apoptosis. After knockdown of CDK4, the level of p21 was detected but the specific regulatory mechanism between CDK4 and p21 warrant further research. The expression of proteins associated with cell cycle progression, invasion and apoptotic cell death in response to miR-486 overexpression or downregulation need to be detected in further research. These are the limitation of this study.

In conclusion, the present study revealed that miR-486 was downregulated in esophageal squamous carcinoma and functions as a tumor suppressor by affecting cell proliferation, colony formation and apoptosis by targeting CDK4 and BCAS2. This is the first study concerning the biological function of miR-486 and its target genes in esophageal squamous 
carcinoma. This novel pathway has the potential for a greater understanding of the pathogenesis of esophageal squamous carcinoma, and drug targets could be developed for the diagnosis and therapeutic treatment of esophageal squamous carcinoma in the future.

\section{References}

1. Beral V and Peto R: UK cancer survival statistics. BMJ 341 (aug11 1): c4112, 2010.

2. Layke JC and Lopez PP: Esophageal cancer: A review and update. Am Fam Physician 73: 2187-2194, 2006.

3. Wu J, Wu X, Liang W, Chen C, Zheng L and An H: Clinicopathological and prognostic significance of chemokine receptor CXCR4 overexpression in patients with esophageal cancer: A meta-analysis. Tumour Biol 35: 3709-3715, 2014

4. Gao YF, Yuan F, Liu J, Li LP, He YC, Gao RJ, Cai YD and Jiang Y: Identification of new candidate Genes and chemicals related to esophageal cancer using a hybrid interaction network of chemicals and proteins. PLoS One 10: e0129474, 2015.

5. van Hagen P, Hulshof MC, van Lanschot JJ, Steyerberg EW, van Berge Henegouwen MI, Wijnhoven BP, Richel DJ, Nieuwenhuijzen GA, Hospers GA, Bonenkamp JJ, et al; CROSS Group: Preoperative chemoradiotherapy for esophageal or junctional cancer. N Engl J Med 366: 2074-2084, 2012.

6. Ming Z, Jiang D, Hu Q, Li X, Huang J, Xu Y, Liu Y, Xu C, Hua X and Hou Y: Diagnostic application of PIK3CA mutation analysis in Chinese esophageal cancer patients. Diagn Pathol 9: 153, 2014

7. Agarwal D, Pineda S, Michailidou K, Herranz J, Pita G, Moreno LT, Alonso MR, Dennis J, Wang Q, Bolla MK, et al; kConFab Investigators; Australian Ovarian Cancer Study Group; GENICA Network; TNBCC: FGF receptor genes and breast cancer susceptibility: Results from the Breast Cancer Association Consortium. Br J Cancer 110: 1088-1100, 2014

8. Hollstein MC, Metcalf RA, Welsh JA, Montesano R and Harris CC: Frequent mutation of the p53 gene in human esophageal cancer. Proc Natl Acad Sci USA 87: 9958-9961, 1990.

9. Zhao H, Zheng L, Li X and Wang L: FasL gene -844T/C mutation of esophageal cancer in South China and its clinical significance. Sci Rep 4: 3866, 2014

10. Meng XR, Lu P, Mei JZ, Liu GJ and Fan QX: Expression analysis of miRNA and target mRNAs in esophageal cancer. Braz J Med Biol Res 47: 811-817, 2014.

11. Berezikov E, Guryev V, van de Belt J, Wienholds E, Plasterk RH and Cuppen E: Phylogenetic shadowing and computational identification of human microRNA genes. Cell 120: 21-24, 2005.

12. Zamore PD and Haley B: Ribo-gnome: The big world of small RNAs. Science 309: 1519-1524, 2005.

13. Pillai RS: MicroRNA function: Multiple mechanisms for a tiny RNA? RNA 11: 1753-1761, 2005.

14. Bartel DP: MicroRNAs: Genomics, biogenesis, mechanism, and function. Cell 116: 281-297, 2004.

15. Esquela-Kerscher A and Slack FJ: Oncomirs - microRNAs with a role in cancer. Nat Rev Cancer 6: 259-269, 2006.

16. Chen CZ, Li L, Lodish HF and Bartel DP: MicroRNAs modulate hematopoietic lineage differentiation. Science 303: 83-86, 2004.

17. Hiyoshi Y, Kamohara H, Karashima R, Sato N, Imamura Y Nagai Y, Yoshida N, Toyama E, Hayashi N, Watanabe M, et al: MicroRNA-21 regulates the proliferation and invasion in esophageal squamous cell carcinoma. Clin Cancer Res 15: 1915-1922, 2009.

18. Feber A, Xi L, Luketich JD, Pennathur A, Landreneau RJ, Wu M, Swanson SJ, Godfrey TE and Litle VR: MicroRNA expression profiles of esophageal cancer. J Thorac Cardiovasc Surg 135: 255-260, discussion 260, 2008.

19. Li B, Xu WW, Han L, Chan KT, Tsao SW, Lee NPY, Law S, $\mathrm{Xu}$ LY, Li EM, Chan KW, et al: MicroRNA-377 suppresses initiation and progression of esophageal cancer by inhibiting CD133 and VEGF. Oncogene 36: 3986-4000, 2017.

20. Gao X, Wang X, Cai K, Wang W, Ju Q, Yang X, Wang H and Wu H: MicroRNA-127 is a tumor suppressor in human esophageal squamous cell carcinoma through the regulation of oncogene FMNL3. Eur J Pharmacol 791: 603-610, 2016.

21. Fu H, Tie Y, Xu C, Zhang Z, Zhu J, Shi Y, Jiang H, Sun Z and Zheng X: Identification of human fetal liver miRNAs by a novel method. FEBS Lett 579: 3849-3854, 2005.
22. Miya K, Shimojima K, Sugawara M, Shimada S, Tsuri H, Harai-Tanaka T, Nakaoka S, Kanegane H, Miyawaki T and Yamamoto T: A de novo interstitial deletion of 8 p11.2 including ANK1 identified in a patient with spherocytosis, psychomotor developmental delay, and distinctive facial features. Gene 506: 146-149, 2012.

23. Oh HK, Tan AL, Das K, Ooi CH, Deng NT, Tan IB, Beillard E, Lee J, Ramnarayanan K, Rha SY, et al: Genomic loss of miR-486 regulates tumor progression and the OLFM4 antiapoptotic factor in gastric cancer. Clin Cancer Res 17: 2657-2667, 2011.

24. Goto K, Oue N, Shinmei S, Sentani K, Sakamoto N, Naito Y, Hayashi T, Teishima J, Matsubara A and Yasui W: Expression of miR-486 is a potential prognostic factor after nephrectomy in advanced renal cell carcinoma. Mol Clin Oncol 1: 235-240, 2013.

25. Huang XP, Hou J, Shen XY, Huang CY, Zhang XH, Xie YA and Luo XL: MicroRNA-486-5p, which is downregulated in hepatocellular carcinoma, suppresses tumor growth by targeting PIK3R1. FEBS J 282: 579-594, 2015.

26. Wang J, Tian X, Han R, Zhang X, Wang X, Shen H, Xue L, Liu Y, Yan X, Shen J, et al: Downregulation of miR-486-5p contributes to tumor progression and metastasis by targeting protumorigenic ARHGAP5 in lung cancer. Oncogene 33: 1181-1189, 2014.

27. Zhang G, Liu Z, Cui G, Wang X and Yang Z: MicroRNA-486-5p targeting PIM-1 suppresses cell proliferation in breast cancer cells. Tumour Biol 35: 11137-11145, 2014.

28. Yi Y, Lu X, Chen J, Jiao C, Zhong J, Song Z, Yu X and Lin B: Downregulated miR-486-5p acts as a tumor suppressor in esophageal squamous cell carcinoma. Exp Ther Med 12: 3411-3416, 2016.

29. Allum, W.H., et al., Guidelines for the management of oesophageal and gastric cancer. Gut, 2011.60(11): p. 1449-72.

30. Jemal A, Siegel R, Ward E, Hao Y, Xu J and Thun MJ: Cancer statistics, 2009. CA Cancer J Clin 59: 225-249, 2009.

31. Trichopoulos D: The unequal burden of cancer. BMJ 320: 321, 2000.

32. Parkin DM, Bray FI and Devesa SS: Cancer burden in the year 2000. The global picture. Eur J Cancer 37 (Suppl 8): S4-S66, 2001.

33. Mandard AM, Hainaut P and Hollstein M: Genetic steps in the development of squamous cell carcinoma of the esophagus. Mutat Res 462: 335-342, 2000.

34. Zen K and Zhang CY: Circulating microRNAs: A novel class of biomarkers to diagnose and monitor human cancers. Med Res Rev 32: 326-348, 2012.

35. Guo J, Wang M and Liu X: MicroRNA-195 suppresses tumor cell proliferation and metastasis by directly targeting BCOX1 in prostate carcinoma. J Exp Clin Cancer Res 34: 91, 2015. https:// doi.org/10.1186/s13046-015-0209-7.

36. Kawano M, Tanaka K, Itonaga I, Ikeda S, Iwasaki T and Tsumura H: microRNA-93 promotes cell proliferation via targeting of PTEN in osteosarcoma cells. J Exp Clin Cancer Res 34: 76, 2015.

37. Esteller M: Non-coding RNAs in human disease. Nat Rev Genet 12: 861-874, 2011.

38. Shao Y, Shen YQ, Li YL, Liang C, Zhang BJ, Lu SD, He YY Wang P, Sun QL, Jin YX, et al: Direct repression of the oncogene CDK4 by the tumor suppressor miR-486-5p in non-small cell lung cancer. Oncotarget 7: 34011-34021, 2016.

39. Weinberg RA: The retinoblastoma protein and cell cycle control. Cell 81: 323-330, 1995.

40. Meyerson M and Harlow E: Identification of G1 kinase activity for cdk6, a novel cyclin D partner. Mol Cell Biol 14: 2077-2086.

41. Matsushime H, Ewen ME, Strom DK, Kato JY, Hanks SK, Roussel MF and Sherr CJ: Identification and properties of an atypical catalytic subunit (p34PSK-J3/cdk4) for mammalian D type G1 cyclins. Cell 71: 323-334, 1992.

42. Kato J, Matsushime H, Hiebert SW, Ewen ME and Sherr CJ: Direct binding of cyclin D to the retinoblastoma gene product $(\mathrm{pRb})$ and $\mathrm{pRb}$ phosphorylation by the cyclin D-dependent kinase CDK4. Genes Dev 7: 331-342, 1993

43. Bates S, Bonetta L, MacAllan D, Parry D, Holder A, Dickson C and Peters G: CDK6 (PLSTIRE) and CDK4 (PSK-J3) are a distinct subset of the cyclin-dependent kinases that associate with cyclin D1. Oncogene 9: 71-79, 1994.

44. Botz J, Zerfass-Thome K, Spitkovsky D, Delius H, Vogt B, Eilers M, Hatzigeorgiou A and Jansen-Dürr P: Cell cycle regulation of the murine cyclin E gene depends on an E2F binding site in the promoter. Mol Cell Biol 16: 3401-3409, 1996. 
45. Sherr CJ and Roberts JM: CDK inhibitors: Positive and negative regulators of G1-phase progression. Genes Dev 13: 1501-1512, 1999.

46. Neumann B, Walter T, Hériché JK, Bulkescher J, Erfle H, Conrad C, Rogers P, Poser I, Held M, Liebel U, et al: Phenotypic profiling of the human genome by time-lapse microscopy reveals cell division genes. Nature 464: 721-727, 2010.

47. Song EJ, Werner SL, Neubauer J, Stegmeier F, Aspden J, Rio D, Harper JW, Elledge SJ, Kirschner MW and Rape M: The Prp19 complex and the Usp4Sart3 deubiquitinating enzyme control reversible ubiquitination at the spliceosome. Genes Dev 24: 1434-1447, 2010.
48. Kittler R, Surendranath V, Heninger AK, Slabicki M, Theis M, Putz G, Franke K, Caldarelli A, Grabner H, Kozak K, et al: Genome-wide resources of endoribonuclease-prepared short interfering RNAs for specific loss-of-function studies. Nat Methods 4: 337-344, 2007.

49. Kuo PC, Tsao YP, Chang HW, Chen PH, Huang CW, Lin ST, Weng YT, Tsai TC, Shieh SY and Chen SL: Breast cancer amplified sequence 2, a novel negative regulator of the p53 tumor suppressor. Cancer Res 69: 8877-8885, 2009.

(i) (2) This work is licensed under a Creative Commons CY NO ND Attribution-NonCommercial-NoDerivatives 4.0 International (CC BY-NC-ND 4.0) License. 